\title{
A Simplified Fermi Accelerator Model Under Quadratic Frictional Force
}

\author{
Danila F. Tavares* \\ Departamento de Física - Universidade Estadual Paulista - UNESP \\ Rua 10, 2527 - Santana - CEP: 13500-230 - Rio Claro - SP - Brazil \\ Edson D. Leonel \\ Departamento de Estatística, Matemática Aplicada e Computação - Universidade Estadual Paulista - UNESP \\ Av. 24A, 1515 - CEP: 13506-900 - Rio Claro - SP - Brazil
}

Received on 23 October, 2007

\begin{abstract}
Some dynamical properties for a simplified version of a one-dimensional Fermi Accelerator Model under the action of a small dissipation is studied. The dissipation is introduced via a damping force which is assumed to be proportional to the square particle's velocity. The dynamics of the model is described by using a twodimensional, nonlinear area contracting mapping for the variables velocity of the particle and time. Our results confirm that the structure of the phase space of the conservative version is replaced by a large number of attracting periodic orbits. For a fixed set of control parameters, we obtain many periodic attractors and show that most of them posses low period. The stable orbits produce a complex structure of basin of attraction whose limit cover almost all phase space, thus suggesting a fractality.
\end{abstract}

Keywords: Fermi Accelerator Model; Damping Forces; Fractal Basin Boundary

\section{INTRODUCTION}

According to previous studies of Enrico Fermi [1], the cosmic rays were primarily accelerated in the interstellar space by collisions of them against moving magnetic fields structures. A dynamical system which corresponds in spirit to Fermi's original model was later proposed by Stanislaw Ulam [2, 3] and it consists of a classical particle confined between two infinitely heavy and rigid walls and therefore suffering elastic collisions with them. One of the walls is assumed to be fixed while the other one is periodically time moving. This model is sometimes referred as to the Fermi-Ulam Model (FUM) [4$6]$ and was studied in many different versions and considering different approaches as well as external fields and damping forces [7-10]. Additionally, this subject has been object of intense study in last years [11-14] and many tools used to characterize such system can be extended to encompass to much more complex billiard problems. One of the approaches commonly used is the well known simplified version presented in [15]. For such a simplified version, it is assumed that both walls are fixed but that, after the collisions with one of the walls, the particle suffers an exchange of energy and momentum as if the wall were moving. This approach allows one substantially to speed up the numerical simulations and moreover such approach retains the nonlinearity of the problem.

There are many different ways to introduce damping forces in the system. One of them is considering inelastic collisions of the particle with one or either walls. Thus, the particle experiences a fractional loss of energy upon collision. As a consequence, the system no longer preserves the phase space measure and its mixed structure is destroyed. Therefore, one can obtain attractors and in particular, chaotic ones. An im-

*Electronic address: danilafterc.unesp.br portant property that can be extracted from the dynamics in the presence of dissipation is the occurrence of a boundary crisis [16]. After the crisis, the chaotic attractor does not exist anymore. It is then replaced by a chaotic transient which is described by a power law [17]. Other different option to introduce damping forces is consider the particle moving in the presence of a drag force. This kind of dissipation acts along the full trajectory of the particle, contrary to the inelastic collisions where it acts only in the instant of the impact. Despite both cases are very common in a real experimental system, the two damping forces yield profound differences in the dynamics of the model.

The main goal of this paper is to characterize the effects of a dissipative force on a simplified FUM that, in our case, is introduced in the system via a relative motion of a particle inside a fluid, like a gas. Recently, Leonel and McClintock [18] had shown that, for a damping force proportional the velocity of the particle and considering a simplified version, the system exhibits a determinant of the Jacobian matrix equals to the unity. Such a result thus confirms that parts of the phase space show the property of area preservation. In our approach however, we assume that the dissipative force is considered to be proportional to the square particle's velocity. Some consequences of this kind of damping force is that the determinant of the Jacobian matrix is no longer the unity, thus the system presents area contraction on the phase space. Additionally, the system has an arbitrarily large number of attracting fixed points and periodic orbits. Such large number of stable orbits yields a complex structure of closely interwoven basins of attraction, whose boundaries fill almost all the phase space. In particular, many observed attractors are characterized by low periods. We must emphasize that studies of nonlinear dynamical systems considering a single mechanical rotor model [19] had shown that, in the Hamiltonian case of the purely rotor map, it exhibits a huge number of stable periodic orbits and each of them turn into an attracting sink when 
a small amount of dissipation is applied. Moreover, it is expected that the complexity of such model should be extended for higher-dimensional systems, as for example a double rotor [20], where it was found many more than 1000 coexisting low-period periodic attractors. We stress that the simplified dissipative FUM is also a suitable model to exhibit a large number of coexisting attractors. Therefore, one of the aims of this paper is to study a qualitative characterization of low periodic attractors and their basins of attraction.

The paper is organized as follows. In section II we describe the model and the procedures used in the construction of the mapping. Section III is devoted to discuss the behavior of the phase space and a classification of the periodic orbits observed, the number of attractors found in the system for a specific range of initial conditions and their basin of attraction. Finally, in section IV we present our conclusions.

\section{THE MODEL AND THE MAPPING}

The Fermi Accelerator Model consists of a classical particle of mass $m$ confined in and bouncing between two parallel, horizontal and rigid walls. One wall is fixed and the other one moves periodically in time. We assume that the particle suffers elastic collisions with either walls. The particle is in the presence of a drag force that we assume to be proportional to the square particle's velocity, i.e. $F=-\eta v^{2}$, where $\eta$ is a drag coefficient. In our approach we will consider only a simplified version of the model (see Ref. [15]). We therefore assume that both walls are fixed, one of them at $x=l$ and the other one at $x=0$. However, when the particle suffers a collision with one of them (say with that at $x=0$ ), it suffers an exchange of energy and momentum as if the wall were moving according to the equation $x_{w}(t)=\varepsilon^{\prime} \cos (\omega t)$. The control parameter $\varepsilon$ denotes the amplitude of oscillation and $\omega$ is the frequency of oscillation. This simplification retains the nonlinearity of the problem and avoids the inconveniency of finding numerical solutions of transcendental equations.

The dynamics of the problem might be described by a two-dimensional nonlinear mapping of the type $T\left(v_{n}, t_{n}\right)=$ $\left(v_{n+1}, t_{n+1}\right)$. Before obtain the equations of the mapping, let us firstly discuss on the initial conditions. We consider that the particle is at the position $x=0$ in the time $t=t_{n}$ with the velocity $v=v_{n}$. Applying the second Newton's law, $\sum F=m a$, we obtain that $-\eta v^{2}=[m d v / d t]$. After solving this equation and considering an elastic reflection of the particle with the fixed wall at $x=l$ and after defining the following dimensionless variables $V_{n}=v_{n} /(\omega l), \delta=\eta l, \varepsilon=\varepsilon^{\prime} / l, \phi_{n}=\omega t_{n}$ the equations that describe the dynamics of the problem are written as

$$
T:\left\{\begin{array}{l}
V_{n+1}=\left|V_{n}^{*}-2 \varepsilon \sin \left(\phi_{n+1}\right)\right| \\
\phi_{n+1}=\phi_{n}+2\left[\frac{e^{\delta}-1}{V_{n} \delta}\right] \bmod (2 \pi)
\end{array}\right.
$$

where the term $V_{n}^{*}=V_{n} /\left(2 e^{\delta}-1\right)$. The modulus function in the equation of velocity was introduced to preserve the particle traveling into the region between the walls. The modulus function has no effect on the motion of the particle if it moves in the positive direction after the collision. It is important emphasize that this approximation is valid only for small values of $\varepsilon$.

After a straightforward algebra, it is easy to show that the determinant of the Jacobian matrix for this version of the problem is given by

$$
\operatorname{det}(J)=\operatorname{sign}\left[V_{n}^{*}-2 \varepsilon \sin \left(\phi_{n+1}\right)\right] \times \frac{1}{\left(2 e^{\delta}-1\right)},
$$

where the function $\operatorname{sign}(u)=1$ if $u>0$ and $\operatorname{sign}(u)=-1$ if $u<0$.

An analysis of the determinant of the Jacobian matrix $(J)$ allow us to conclude that the system shrinks area of the phase space for values of $\delta \neq 0$.

\section{NUMERICAL RESULTS}

\section{A. The Phase Space}

Let us discuss in this section our numerical results. The introduction of a damping force yields drastic changes in the phase space while it is compared to the non dissipative version of the model. It is well known that for the conservative case, the phase space shows a chaotic sea surrounding KAM islands and a set of invariant spanning curves limiting the energy gain of a bouncing particle (see for example [11]). Such a structure is totally destroyed in the presence of quadratic frictional force. To illustrate such a destruction, it is shown in Fig. 1 the corresponding phase espace for a simplified version of a dissipative version of the FUM under quadratic frictional force. Figure 1(a) shows the attracting periodic orbits while

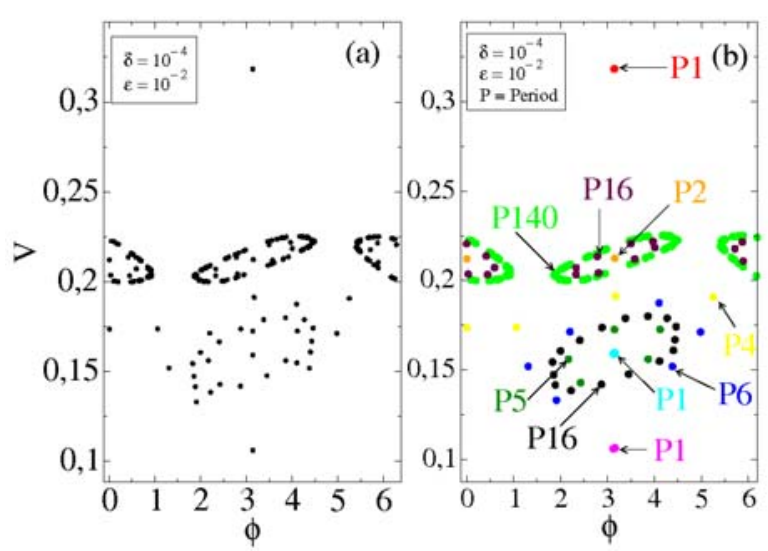

FIG. 1: (Colour online) (a) Phase space for a dissipative version of a Simplified Fermi Accelerator Model. We have used $\varepsilon=10^{-2}$ and $\delta=10^{-4}$. (b) Classification of the periodic orbits observed in (a).

their classification is shown in Fig. 1(b). It is easy to see that the phase space for this version of the model presents a large number of attracting fixed points (sinks), whose orbits are plotted in different colours. We must say that the number 
of attractors depends on the values of the control parameters used as well as on the grid of initial conditions taken into account. The results shown in Fig. 1 were obtained using the following set of control parameters: $\varepsilon=10^{-2}$ and $\delta=10^{-4}$. We have considered a grid of $7 \times 10$ initial conditions for the intervals $\phi_{0} \in(0,2 \pi]$ and $V_{0} \in[\varepsilon, 0.5]$ respectively. Moreover, each initial condition was evolved up to $10^{6}$ iterations as an attempt to avoid the transient effects. For this set of fixed control parameters, the highest periodic orbit obtained has period 140 (shown in green) and the lower periodic orbits found exhibits period 1 (those shown in red, cyan and magenta in Fig. $1(\mathrm{~b})$ ).

\section{B. The Number of Attractors}

Generally, dissipative nonlinear dynamical systems often exhibit a rich and varied behavior including a large number of attractors. As an attempt to estimate the number of coexisting periodic orbits in the present model, we have made two different simulations considering different grids of initial conditions. Firstly we have considered $10^{4}$ different initial conditions, i.e. a grid of $100 \times 100$ uniformely distributed in the interval $\phi \in(0,2 \pi]$ and $V \in[\varepsilon, 0.5]$. Secondly, we have evolved a simulation for $25 \times 10^{4}$ initial conditions on a grid of $500 \times 500$ for the same interval of $V \times \phi$. We have used a particular set of control parameters $\varepsilon=10^{-2}$ and $\delta=10^{-4}$. It must be emphasized that each initial condition for both simulations was evolved for a long run of $10^{7}$ iterations, thus avoiding transient effects. It is shown in Fig. 2 a histogram of frequency plotted in logarithmic scale. It is easy to see that low

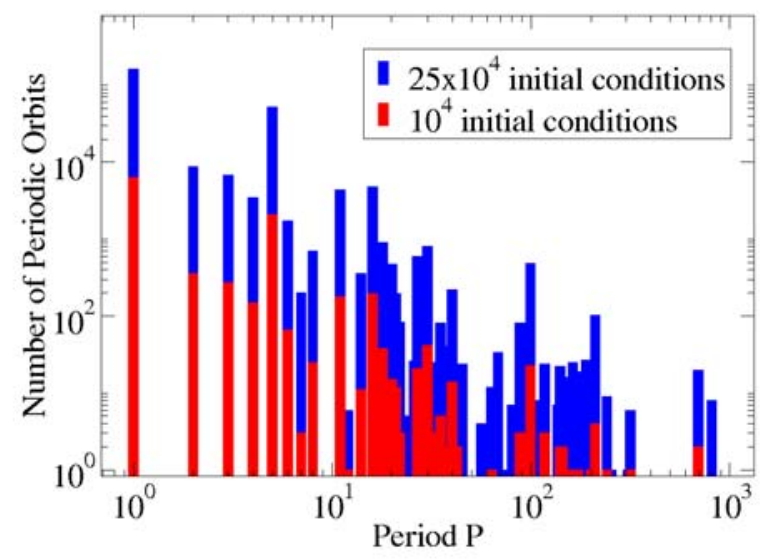

FIG. 2: (Colour online) Number of periodic orbits and their period for a Simplified Fermi Accelerator Model (dissipative version) using $\varepsilon=10^{-2}$ and $\delta=10^{-4}$. The red columns shows the results for $10^{4}$ initial conditions while the blue columns were obtained for $25 \times 10^{4}$ initial conditions.

periodic attractors (those with period $<10$ ) dominate over the high periodic ones, thus stable orbits of high period are rarely found. It is worth mentioning that the number of attractors obtained depends on the grid of the initial conditions used. For
$10^{4}$ initial conditions, the highest periodic orbit found was 693 whereas for $25 \times 10^{4}$ initial conditions we detect an orbit of period 810. As larger the grid of initial conditions used, larger the number of attractors will be found in the system.

\section{The Basin of Attraction}

We now present the basin of attraction for the attracting periodic orbits. It is shown in Fig. 3 the basins of attraction for the fixed points shown in Fig. 1. We can see that the largest

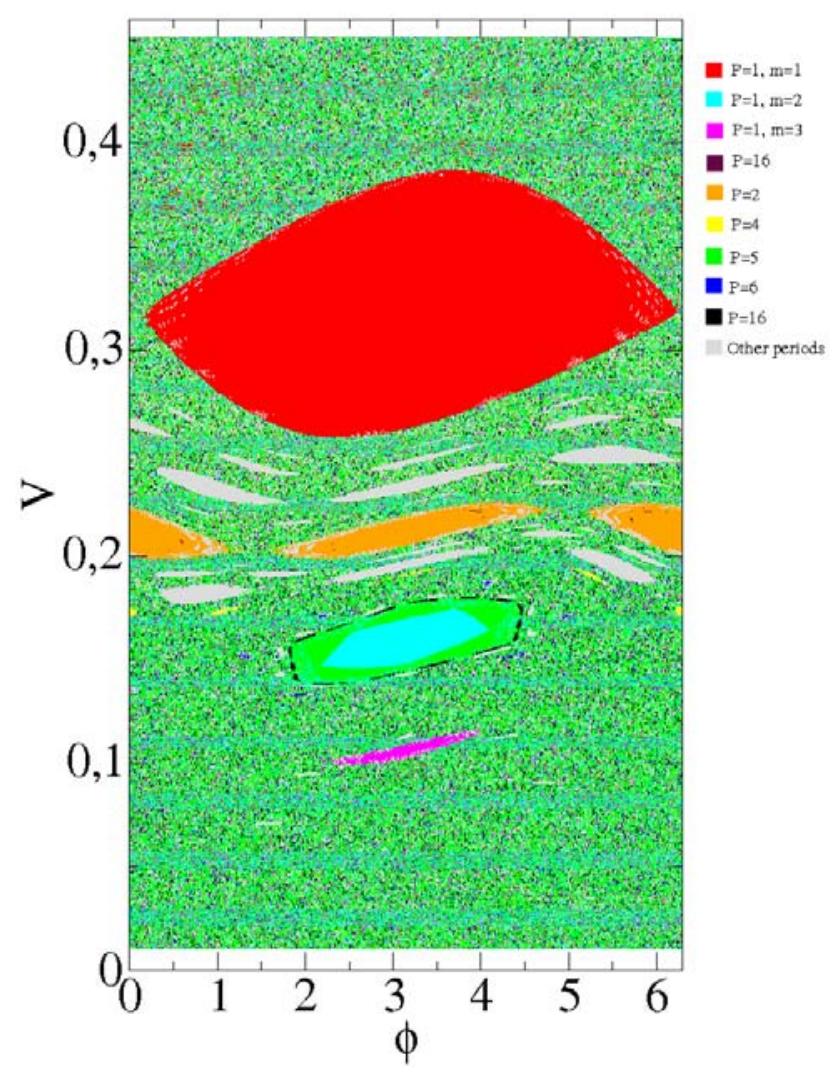

FIG. 3: (Colour online) Basins of attraction for the periodic attractors indicated in the Fig. 1. We have iterated $10^{6}$ initial conditions considering a grid of $1000 \times 1000$ for the ranges $\phi \in(0,2 \pi]$ and $V \in[\varepsilon, 0.5]$. The control parameters used were $\varepsilon=10^{-2}$ and $\delta=10^{-4}$.

basin of attraction (shown in red) corresponds to the main orbit of the phase space. It is indeed of the period 1 family $\left(P_{1}\right)$. In general, all the basins of $P_{1}$ orbits (those shown in cyan and magenta in Fig. 3) are relatively large as compared to those of other period. We must say that the basins of attraction for high periodic orbits require a larger grid of resolution on the plane of initial conditions. The control parameters used in the construction of the basins were $\varepsilon=10^{-2}$ and $\delta=10^{-4}$. We have used a range of $V \in[\varepsilon, 0.5]$ and $\phi \in(0,2 \pi]$. Both ranges were divided in 1000 parts each, leading to a total of $10^{6}$ dif- 
ferent initial conditions. Moreover, each initial condition was iterated up to $10^{6}$ times.

In systems with many coexisting attractors as found here, the structure of their basins is very complex. We expect that such complexity in the closely related basins of attraction corresponds to typical properties of systems with a large number of coexisting attractors.

\section{CONCLUSIONS}

We have studied a one-dimensional Fermi Accelerator Model in the presence of a frictional force proportional to the square particle's velocity. Our results confirm that the system experiences contraction of the phase space area and shows a large number of coexisting periodic attractors. We obtain the number of periodic attractors for specific ranges of initial conditions and fixed control parameters. We note that the systems with many attractors produce a structure of basin of attraction very complex. Our results suggest that, instead of turning to a basin boundary smooth, the addition of small amount of dissipation leads to an apparently fractal basin boundary.

\section{Acknowledgments}

DFT thanks to Fundação de Amparo à Pesquisa do Estado de São Paulo - FAPESP, Brazilian agency. EDL gratefully acknowledges support from FAPESP, CNPq and FUNDUNESP, Brazilian agencies.
[1] E. Fermi, Phys. Rev. 75, 1169 (1949).

[2] S. Ulam. Proc. 4th Berkeley Symposium on Math, Statistics and Probability, Vol. 3 (Berkeley, CA: California University Press), p. 315 (1961).

[3] L. D. Pustyl'nikov, Theor. Math. Phys., 57, 1035 (1983).

[4] M. A. Lieberman and A. J. Lichtenberg, Phys. Rev. A, 5, 1852 (1972).

[5] L. D. Pustylnikov, Doklad. A. Nau. SSSR, 241, 1035 (1978).

[6] L. D. Pustyl'nikov, Soviet Math. Dokl. 35, 1 (1987)

[7] A. J. Lichtenberg, M. A. Lieberman, and R. H. Cohen, Physica D 1, 291 (1980).

[8] M. A. Lieberman and K. Y. Tsang, Phys. Rev. Lett. 55, 908 (1985).

[9] T. Krüger, L. D. Pustyl'nikov, and S. E. Troubetzkoy, Nonlinearity 8, 397 (1995).

[10] E. D. Leonel, P. V. E. McClintock, and J. K. L. da Silva, Phys. Rev. Lett. 93, 014101(1-4) (2004).

[11] E. D. Leonel, J. K. L. da Silva, and S. O. Kamphorst, Physica A 331, 435 (2004).
[12] D. G. Ladeira and E. D. Leonel, Chaos 17, 013119 (2007).

[13] J. K. L. da Silva, D. G. Ladeira, E. D. Leonel, P. V. E. McClintock, and S. O. Kamphorst, Braz. J. Phys. 36, 700 (2006).

[14] R. E. de Carvalho, F. C. de Souza, and E. D. Leonel, J. Phys. A: Math and Gen. 39, 3561 (2006).

[15] A. J. Lichtenberg and M. A. Lieberman, Regular and Chaotic Dynamics, Appl. Math. Sci., 38, Springer-Verlag, New York (1992).

[16] E. D. Leonel and P. V. E. McClintock, J. Phys. A: Math. and Gen. 38, L425 (2005).

[17] E. D. Leonel and R. E. de Carvalho, Phys. Lett. A 364, 475 (2007).

[18] E. D. Leonel and P. V. E. McClintock, J. Phys. A 39, 11399 (2006).

[19] U. Feudel, C. Grebogi, B. R. Hunt, and J. A. Yorke, Phys. Rev. E 54, 1 (1996).

[20] U. Feudel, C. Grebogi, L. Poon, and J. A. Yorke, Chaos, Solitons and Fractals, 9, 171 (1998). 